\title{
Limit of the Solution of a PDE in the Degenerate Case*
}

\author{
Alassane Diedhiou \\ Département de Mathématiques, Université de Ziguinchor, Ziguinchor, Senegal \\ Email: asana@ucad.sn
}

Received May 21, 2012; revised January 10, 2013; accepted January 17, 2013

\begin{abstract}
In this paper we show that we can have the same conclusion for the limit of the solution $u^{\epsilon, \delta}$ if we suppose the case of hypoellipticity.
\end{abstract}

Keywords: Homogenization; Large Deviations Principle; Stochastic Differential Equations

\section{Introduction}

Let us consider the parabolic PDE:

$$
\left\{\begin{array}{l}
\frac{\partial u^{\epsilon, \delta}}{\partial t}(t, x)=L_{\epsilon, \delta} u^{\epsilon, \delta}(t, x)+\frac{1}{\epsilon} f\left(\frac{x}{\delta}, u^{\epsilon, \delta}(t, x)\right) \\
u^{\epsilon, \delta}(0, x)=g(x), x \in \mathbb{I R}^{\mathrm{d}}
\end{array}\right.
$$

We study in this paper the behavior of $u^{\epsilon, \delta}$ when $\epsilon, \delta$ tend to zero, and $\lim _{\epsilon, \delta \downarrow 0} \frac{\delta}{\epsilon}=0$. We suppose that the matrix of the second order coefficients of $L_{\epsilon, \delta}$ is degenerate, in fact we formulate here a hypoellipticity condition of Hörmander type (see e.g. David Nualart [1]). Diédhiou and Manga in [2] studied the limit of $u^{\epsilon, \delta}$ with a nondegenerate condition of the matrix. In Freidlin \& Sowers[3], three cases are considered, with the assumption that the matrix is non, but we formulate here a hypoellipticity condition of. Since the parameter $\delta$ (homogeneization parameter) decreases quickly than $\epsilon$ (large deviations principle parameter) to zero we must homogenize first and apply the large deviations principle.

We use essentially probabilistic tools to solve our problem.

Let $\left(\Omega, \mathcal{F},\left(\mathcal{F}_{t}\right)_{t \geq 0}\right)$ a probability filtered space. We consider the $\operatorname{IR}^{\mathrm{d}}(d \geq 1)$ valued process $\left(X_{t}^{x, \epsilon, \delta}\right)$ solution of the SDE:

$$
\left\{\begin{array}{l}
\mathrm{d} X_{t}^{\chi, \epsilon, \delta}=\sqrt{\epsilon} \sigma\left(\frac{X_{t}^{x, \epsilon, \delta}}{\delta}\right) \mathrm{d} W_{t}+B^{\epsilon, \delta}\left(\frac{X_{t}^{\chi, \epsilon, \delta}}{\delta}\right) \mathrm{d} t \\
X_{0}^{x, \epsilon, \delta}=x
\end{array}\right.
$$

where $\sigma: \mathrm{IR}^{\mathrm{d}} \rightarrow \mathrm{IR}^{\mathrm{d} \times d}, B^{\varepsilon, \delta}: \mathrm{IR}^{\mathrm{d}} \rightarrow \mathrm{IR}^{\mathrm{d}}$, and $\left\{W_{t} ; t \geq 0\right\}$ is a d-dimensional standard Brownian mo-

*This work was supported, in part, by grants from FIRST (Fonds d'Impulsion pour la Recherche Scientique et Technique). tion.

We assume that $\sigma$ and $B^{\epsilon, \delta}$ are smooth mappings from $\mathrm{IR}^{\mathrm{d}}$, and periodic with period one in each direction.

The mapping $B^{\epsilon, \delta}$ is assumed to be of the form :

$$
B^{\epsilon, \delta}=\frac{\epsilon}{\delta} B_{0}+B_{1}+B_{2}^{\epsilon, \delta}
$$

where $B_{0}, B_{1}$ and $B_{2}^{\epsilon, \delta}$ are in $\mathcal{C}^{\infty}\left(\mathrm{IR}^{\mathrm{d}}, \mathrm{IR}^{\mathrm{d}}\right)$ for every $\epsilon>0, \delta>0$ and

$$
\lim _{\epsilon, \delta \downarrow 0}\left\|B_{2}^{\epsilon, \delta}\right\|_{\mathcal{C}_{p}\left(\mathbb{R}^{\mathrm{d}}, \mathbb{R}^{\mathrm{d}}\right)}=0,
$$

where $C_{p}\left(\operatorname{IR}^{\mathrm{d}}, \mathrm{IR}^{\mathrm{d}}\right)$ be the collection of periodic continuous mappings from $\mathrm{IR}^{\mathrm{d}}$ into $\mathrm{IR}^{\mathrm{d}}$.

The infinitesimal generator $L_{\epsilon, \delta}$ is gigen by

$$
L_{\epsilon, \delta}=\frac{\epsilon}{2} \sum_{i, j=1}^{d} a_{i j}\left(\frac{x}{\delta}\right) \frac{\partial^{2}}{\partial x_{i} \partial x_{j}}+\sum_{i=1}^{d} B_{i}^{\epsilon, \delta}\left(\frac{x}{\delta}\right) \frac{\partial}{\partial x_{i}}
$$

and where $g \in \mathcal{C}\left(\mathrm{IR}^{\mathrm{d}}, \mathrm{IR}^{+}\right)$is a bounded function and we set

$$
\sup _{x \in \mathbb{R}^{d}} g(x)=\bar{g}<\infty .
$$

Let set

$$
G_{0}=\left\{x \in \mathrm{IR}^{\mathrm{d}}: g(x)>0\right\}
$$

since $g$ is continuous we have $\overline{G_{0}}=\overline{G_{0}}$.

We assume that $f$ is periodic in each direction, with respect to the first argument, and it verifies:

- $\forall x \in \mathbb{I R}^{\mathrm{d}}, f(x, 1)=0$

- There exists $C \in \mathcal{C}\left(\operatorname{IR}^{\mathrm{d}} \times \operatorname{IR}, \mathrm{IR}\right)$ bounded such that

with

$$
f(x, y)=C(x, y) \cdot y
$$

$$
\begin{aligned}
& \cdot C(x, y)>0, \forall x \in \mathbb{I R}^{d}, y \in[0,1) \\
& \cdot C(x, y) \leq 0, \forall x \in \mathbb{I}^{d}, y>1
\end{aligned}
$$


and we assume that

$$
\max _{y \in \mathbb{R}} C(x, y)=C(x)=C(x, 0)>0, \forall x \in \mathbb{I R}^{\mathrm{d}} .
$$

Let us consider the progressive measurable process $\left(Y^{\epsilon, \delta, t, x}, Z^{\epsilon, \delta, t, x}\right)$ solution of the BSDE:

$$
\left\{\begin{array}{l}
Y_{s}^{\epsilon, \delta, t, x}=g\left(X_{t}^{\epsilon, \delta, t, x}\right)+\frac{1}{\epsilon} \int_{s}^{t} f\left(\frac{X_{r}^{\epsilon, \delta, t, x}}{\delta}, Y_{r}^{\epsilon, \delta, t, x}\right) \mathrm{d} r \\
-\frac{1}{\sqrt{\epsilon}} \int_{s}^{t} Z_{r}^{\epsilon, \delta, t, x} \mathrm{~d} W_{r}, 0 \leq s \leq t, \\
\mathrm{IE}\left[\int_{0}^{t}\left|Z_{r}^{\epsilon, \delta, t, x}\right|^{2} \mathrm{~d} r\right]<\infty .
\end{array}\right.
$$

By Pardoux and Peng [4], we have for all

$$
\begin{gathered}
(t, x) \in\left[0,+\infty\left[\times \mathrm{IR}^{\mathrm{d}},\right.\right. \\
u^{\epsilon, \delta}(t, x)=Y_{0}^{\epsilon, \delta, t, x} .
\end{gathered}
$$

The matrix $a=\sigma \sigma^{*}$ (where $(*)$ is the symbol of transposition) is degenerate. Let us consider the

Definition 1.1 The Lie bracket between the vector fields $A_{j}$ and $A_{k}$ is defined by

$$
\left[A_{j}, A_{k}\right]=A_{j}^{\nabla} A_{k}-A_{k}^{\nabla} A_{j},
$$

where $A_{j}^{\nabla} A_{k}=A_{j}^{\ell} \partial_{\ell} A_{k}^{i} \frac{\partial}{\partial x_{i}}$.

We assume that the matrix $\sigma$ of the column vectors $\sigma_{j}$ verifies the strong Hörmander condition, defined by the

Definition 1.2 Let $H(n, x)$ be the set of Lie brackets of $\left(\sigma_{j}(x)\right)_{1 \leq j \leq d}$ of order lower than $n$ at the point $x \in \mathrm{IR}^{\mathrm{d}}$.

We say that the matrix $\sigma$ satisfies the strong Hörmander condition (called SHC) if for all $x \in \mathrm{IR}^{\mathrm{d}}$, there exists $n_{x} \in \mathrm{IN}$ such that $H\left(n_{x}, x\right)$ generates $\mathrm{IR}^{\mathrm{d}}$.

We organize this paper as follows. Section 2 contains the results of large deviations principle. In Section 3 we study the behavior of the solution of the PDE (1).

\section{Large Deviations Principle}

Since, $\lim _{\epsilon \downarrow 0} \frac{\delta_{\epsilon}}{\epsilon}=0$ (when we set $\delta=\delta_{\epsilon}$ ) we have a problem of homogenization because the matrix $a(x)=\sigma \sigma^{*}(x)$ is not elliptic.

Since $\delta_{\epsilon}$ tends to zero faster than $\epsilon$, the homogenization dominates, and the large deviations principle will be applied to the problem with constant coefficients.

For the homogeneization in the hypoellipticy case, we use the results of Diédhiou and Pardoux [5] and Pardoux
$[4,6,7]$.

Setting: $\quad \tilde{X}_{t}^{x, \epsilon, \delta_{\epsilon}}=\frac{1}{\delta_{\epsilon}} X_{\left(\frac{\delta_{\epsilon}}{\sqrt{\epsilon}}\right)^{2} t}^{x, \delta_{\epsilon}}$, we have

$$
\left\{\begin{array}{l}
\mathrm{d} \tilde{X}_{t}^{x, \epsilon, \delta_{\epsilon}}=\frac{\delta_{\epsilon}}{\varepsilon} B^{\epsilon, \delta_{\epsilon}}\left(\tilde{X}_{t}^{x, \epsilon, \delta_{\epsilon}}\right) \mathrm{d} t+\sigma\left(\tilde{X}_{t}^{x, \epsilon, \delta_{\epsilon}}\right) \mathrm{d} \tilde{W}_{t}^{\epsilon, \delta_{\epsilon}} \\
\tilde{X}_{0}^{x, \epsilon, \delta_{\epsilon}}=\frac{X}{\delta_{\epsilon}}
\end{array}\right.
$$

where $\left\{\tilde{W}_{t}^{\epsilon, \delta_{\epsilon}}: t \geq 0\right\}$ is a standard Brownian motion.

The $\mathbf{T}^{\mathrm{d}}$-valued process $\tilde{X}_{t}^{x, \epsilon, \delta_{\epsilon}}$, is a Feller process, then has a unique invariant measure $\mu_{\epsilon}$, and we have $\mu_{\epsilon} \Rightarrow \mu_{0}$, when $\epsilon \rightarrow 0$ see [5].

We assume that

$$
\int_{\mathbf{T}^{\mathrm{d}}} B_{0}(\mathrm{z}) \mu_{0}(\mathrm{~d} z)=0,
$$

and the homogenized coefficients see [3] are

$$
\begin{aligned}
& \bar{B}=\int_{\mathbf{T}^{\mathrm{d}}}\left(I+\nabla \hat{B}_{0}\right) B_{1}(x) \mu_{0}(\mathrm{~d} x) ; \\
& \bar{A}=\int_{\mathbf{T}^{\mathrm{d}}}\left(I+\nabla \hat{B}_{0}\right) \sigma \sigma^{*}\left(I+\nabla \hat{B}_{0}\right)^{*}(x) \mu_{0}(\mathrm{~d} x) .
\end{aligned}
$$

Let us define, for each $T>0$ and $x \in \mathrm{IR}^{\mathrm{d}}$,

$$
\begin{aligned}
& g_{T, x}^{\epsilon}(\theta)=\epsilon \log \operatorname{IE}\left[\exp \left[\frac{1}{\epsilon}\left\langle\theta, X_{T}^{x, \epsilon, \delta_{\epsilon}}\right\rangle\right]\right], \\
& \epsilon>0, \theta \in \mathrm{IR}^{\mathrm{d}} .
\end{aligned}
$$

We have

$$
\begin{aligned}
g_{T, x}(\theta) & =\lim _{\epsilon \downarrow 0} g_{T, x}^{\epsilon}(\theta) \\
& =\langle\theta, x\rangle+T\left(\frac{1}{2}\langle A \theta, \theta\rangle+\left\langle\bar{B}_{1}, \theta\right\rangle\right), \theta \in \mathbb{I R}^{\mathrm{d}} .
\end{aligned}
$$

The details of the calculation of this limit are the same as in Freidlin and Sowers [3].

In order to establish a large deviations principle, we will consider the

Theorem 2.1 ([8]) Fix $T>0$ and $x \in \mathrm{IR}^{\mathrm{d}}$. Assume that

1) For each $\theta \in \mathrm{IR}^{\mathrm{d}}, g_{T, x}(\theta)$ is well-defined in $[-\infty,+\infty]$.

2) The origin is in the interior of the set

$$
\left\{\theta \in \mathrm{IR}^{\mathrm{d}}: g_{T, x}(\theta)<\infty\right\} \text {. }
$$

3) The set $A=\left\{\theta \in \operatorname{IR}^{d}:\left|g_{T, x}(\theta)\right|<\infty\right\}$ has a nonempty interior $\stackrel{\circ}{A}, \nabla g_{T, x}(\theta)$ is well-defined for all $\theta \in \stackrel{\circ}{A}$, and

$$
\lim _{\theta \rightarrow \partial A, \theta \in A,} \sup \left\|\nabla_{\theta} \boldsymbol{g}_{T, x}(\theta)\right\|=\infty .
$$

Then the random variables $\left\{X_{T}^{x, \epsilon, \delta_{\epsilon}}: \epsilon>0\right\}$ satisfy a 
large deviations principle with rate function $I_{T, x}^{1}$ defined by

$$
\begin{aligned}
& I_{T, x}^{1}(z)=\sup _{\theta \in \mathbb{R}^{\mathrm{d}}}\left\{\langle\theta, z\rangle-g_{T, x}(\theta)\right\}, \\
& z \in \mathbb{I R}^{\mathrm{d}} .
\end{aligned}
$$

The limit $g_{T, x}(\theta)$ satisfies the conditions 1) and 2). For the condition 3 ) we may assume more that the matrix $\bar{A}$ is strictly positive-definite. In fact it is not a strong assumption, for

Example 2.2 If we choose $\mathrm{d}=3, B_{0}=0$ and

$$
\sigma(x, y, z)=\left(\begin{array}{cc}
1 & 0 \\
0 & \sin (2 \pi x) \\
0 & \cos (2 \pi x)
\end{array}\right)
$$

this matrix satisfies the Hörmander condition, and

$$
\sigma \sigma^{*}(x, y, z)=\left(\begin{array}{ccc}
1 & 0 & 0 \\
0 & \frac{1-\cos (4 \pi x)}{2} & \frac{1}{2} \sin (4 \pi x) \\
0 & \frac{1}{2} \sin (4 \pi x) & \frac{1+\cos (4 \pi x)}{2}
\end{array}\right) .
$$

The invariant measure $\mu_{0}$ has the density

$$
p(x, y, z)=2 x \mathrm{II}_{\mathbf{T}^{\mathrm{d}}}(x, y, z) .
$$

Then we have

$$
\bar{A}=\left(\begin{array}{ccc}
1 & 0 & 0 \\
0 & \frac{1}{2} & -\frac{1}{4 \pi} \\
0 & -\frac{1}{4 \pi} & \frac{1}{2}
\end{array}\right) .
$$

Let us consider

$$
\mathcal{J}^{1}(\theta)=\sup _{\theta^{\prime} \in \mathbb{R}^{\mathrm{d}}}\left\{\left\langle\theta, \theta^{\prime}\right\rangle-\overline{\mathcal{J}}^{1}\left(\theta^{\prime}\right)\right\}, \theta \in \mathbb{R}^{\mathrm{d}},
$$

by the assumption on $\bar{A}$, we get

$$
\mathcal{J}^{1}(\theta)=\frac{1}{2}\left\langle\bar{A}^{-1}(\theta-\bar{B}), \theta-\bar{B}\right\rangle, \theta \in \mathrm{IR}^{\mathrm{d}} .
$$

Thus the form of $\mathcal{J}^{1}$ and the assumption on $\bar{A}$ imply that 3 ) is true.

We have the

Theorem 2.3 (Freidlin and Sowers [3]) Fix $T_{0}>0$ and assume that the assumption (3) is true. For every $x \in \mathbb{I R}^{\mathrm{d}}$ and $0<T \leq T_{0}$, the family $\left\{X_{T}^{x, \epsilon, \delta}: \epsilon>0\right\}$ of $\mathrm{IR}^{\mathrm{d}}$ - valued random variables satisfies a large deviations principle $(L D P)$ with rate function

$$
I_{T, x}^{1}(z)=T \mathcal{J}^{1}\left(\frac{z-x}{T}\right), z \in \operatorname{IR}^{\mathrm{d}} .
$$

Furthermore, this $L D P$ is uniform for all $0<T \leq T_{0}$ and $x \in \mathrm{IR}^{\mathrm{d}}$.

Proof: See Freidlin and Sowers [3].

Let us consider some definitions:

$$
S_{0 T}^{1}(\varphi)= \begin{cases}\int_{0}^{T} \mathcal{J}^{1}(\dot{\varphi}(s)) \mathrm{d} s, \text { if is absolutely continuous and } \varphi(0)=x \\ \infty, & \text { if not }\end{cases}
$$

Since the function $\mathcal{J}^{1}$ is convex we can show that

$$
\inf _{\left\{\varphi \in \mathcal{C}^{1}\left([0, T] ; \mathbb{R}^{\mathrm{d}}\right), \varphi(0)=x, \varphi(T)=y\right.} \int_{0}^{T} \mathcal{J}^{1}(\dot{\varphi}(s)) \mathrm{d} s=T \mathcal{J}^{1}\left(\frac{y-x}{T}\right) .
$$

So we have the

Theorem 2.4 For all $T>0$, we assume that the assumption (3) holds. The family

$$
\left\{X_{t}^{x, \epsilon, \delta_{\epsilon}} ; 0 \leq t \leq T ; \epsilon>0\right\},
$$

of $\mathcal{C}\left([0, T] ; \mathrm{IR}^{\mathrm{d}}\right)$-valued random variables satisfies a Large Deviations Principle (LDP) with rate function $S_{0 T}^{1}(\varphi)$ for all $\varphi \in \mathcal{C}\left([0, T] ; \mathbb{I R}^{\mathrm{d}}\right)$.

Proof: See Freidlin and Sowers [3]

\section{Asymptotic Behavior of $\boldsymbol{u}^{\epsilon, \delta_{\epsilon}}$}

We want to apply the technics used by [6], so we consider now the BSDE:

$$
\left\{\begin{array}{l}
Y_{s}^{x, t, \epsilon, \delta_{\epsilon}}=g\left(X_{t}^{x, \epsilon, \delta_{\epsilon}}\right)+\frac{1}{\epsilon} \int_{s}^{t} f\left(\frac{X_{r}^{x, \epsilon, \delta_{\epsilon}}}{\delta_{\epsilon}}, Y_{r}^{X, \epsilon, \delta_{\epsilon}}\right) \mathrm{d} r \\
-\frac{1}{\sqrt{\epsilon}} \int_{s}^{t} Z_{r}^{x, \epsilon, \delta_{\epsilon}} \mathrm{d} W_{r}, 0 \leq s \leq t \\
\operatorname{IE}\left[\int_{0}^{t}\left|Z_{r}^{x, \epsilon, \delta_{\epsilon}}\right|^{2} \mathrm{~d} r\right]<\infty
\end{array}\right.
$$

We know that for all $(t, x) \in\left[0,+\infty\left[\times \mathrm{IR}^{\mathrm{d}}\right.\right.$, the solution $u^{\epsilon, \delta_{\epsilon}}(t, x)$ of the PDE is of the form:

$$
u^{\epsilon, \delta_{\epsilon}}(t, x)=Y_{0}^{x, \epsilon, \delta_{\epsilon}, t},
$$

and by the Feynman-Kac formula, we have

$$
Y_{0}^{X, \epsilon, \delta_{\epsilon}}=\operatorname{IE}\left[g\left(X_{t}^{X, \epsilon, \delta_{\epsilon}}\right) \exp \left\{\frac{1}{\epsilon} \int_{0}^{t} C\left(\frac{X_{r}^{X, \epsilon, \delta_{\epsilon}}}{\delta_{\epsilon}}, Y_{r}^{X, \epsilon, \delta_{\epsilon}}\right) \mathrm{d} r\right\}\right] .
$$

Our aim is to study the behavior of the $u^{\epsilon, \delta_{\epsilon}}(t, x)$ 
when $\epsilon$ tends to zero.

\section{Remark 3.1}

- If $\bar{g} \leq 1$, then $\forall x \in \mathrm{IR}^{\mathrm{d}}, \forall \epsilon>0$,

$$
0 \leq Y_{s}^{x, t, \epsilon, \delta_{\epsilon}} \leq 1, \mathrm{dIP} \times \mathrm{d} s \text { as }
$$

- In the other cases, if

$$
\left.C(x, y) \leq \alpha(y)<0,(x, y) \in \operatorname{IR}^{\mathrm{d}} \times\right] 1,+\infty[
$$

where $\alpha$ is Lipschitz continuous, then

$$
\limsup Y_{0}^{x, t, \epsilon, \delta_{\epsilon}} \leq 1
$$

uniformly in any compact set of $] 0,+\infty\left[\times \mathrm{IR}^{\mathrm{d}}\right.$.

We give the

Definition 3.2 A functional $\tau: C\left([0, t], \mathrm{IR}^{\mathrm{d}}\right) \rightarrow[0, t]$ is a stopping time if for all $\varphi, \tilde{\varphi} \in C\left([0, t], \mathrm{IR}^{\mathrm{d}}\right)$ and all $s \in[0, t], \varphi_{r}=\tilde{\varphi}_{r}$ for all $r \in[0, s]$ and $\tau(\varphi)<s$ imply $\tau(\tilde{\varphi})=\tau(\varphi)$.

Let us set $\Sigma_{t}$ the set of stopping times and $\Theta_{t}$ the set of elements $\tau$ of $\Sigma_{t}$ such that there exists $\mathcal{O}$ such that for all

$$
\varphi \in C\left(\left[0, \infty\left[, \operatorname{IR}^{\mathrm{d}}\right): \tau(\varphi)=\inf \left\{s \leq t:\left(t-s, \varphi_{s}\right) \in \mathcal{O}\right\}\right.\right.
$$

with the convention inf $\varnothing=t . \quad \tau$ is hence a well defined element of $\Sigma_{t}$ and $\mathcal{O}$ is the open set associated.

$$
\tau:\left[0,+\infty\left[\times C\left(\left[0,+\infty\left[, \mathrm{IR}^{\mathrm{d}}\right) \rightarrow[0,+\infty[\right.\right.\right.\right.
$$

is an element of $\Sigma$ (resp. $\Theta$ ) if and only if, for all $t \geq 0, \tau_{t}=\tau(t, \cdot) \in \Sigma_{t} \quad\left(\right.$ resp. $\left.\Theta_{t}\right)$ where

$$
\tau(t, \varphi)=\tau_{t}\left(\varphi_{[0, t]}\right) .
$$

Let us consider the function $V^{*}(t, x)$ defined in ] $0,+\infty\left[\times \operatorname{IR}^{\mathrm{d}}\right.$ by,

$$
\begin{aligned}
& V^{*}(t, x) \\
& =\inf _{\tau \in \Theta_{t}} \sup _{\varphi}\left\{R_{0 \tau}(\varphi), \varphi_{0}=x, \varphi_{t} \in G_{0}, \varphi \in \mathcal{C}([0, t])\right\}
\end{aligned}
$$

where

$$
R_{0 \tau}(\varphi)=\bar{C} \tau-S_{0 \tau}(\varphi), \bar{C}=\int_{\mathbf{T}^{\mathrm{d}}} C(x) \mu_{0}(\mathrm{~d} x) .
$$

Let $\mathcal{M}$ and $\mathcal{E}$ be a partition of $\mathrm{IR}^{+} \times \mathrm{IR}^{\mathrm{d}}$,

$$
\begin{aligned}
& \mathcal{M}=\left\{(t, x) \in \mathrm{IR}^{+} \times \mathrm{IR}^{\mathrm{d}} ; V^{*}(t, x)=0\right\} ; \\
& \mathcal{E}=\left\{(t, x) \in \mathrm{IR}^{+} \times \operatorname{IR}^{\mathrm{d}} ; V^{*}(t, x)<0\right\} .
\end{aligned}
$$

We have

$$
\begin{aligned}
& u^{\epsilon, \delta_{\epsilon}}(t, x) \\
& =\operatorname{IE}\left[g\left(X_{t}^{\epsilon, x, \delta_{\epsilon}}\right) \exp \left\{\frac{1}{\epsilon} \int_{0}^{t} C\left(\frac{X_{r}^{X, \epsilon, \delta_{\epsilon}}}{\delta_{\epsilon}}, Y_{r}^{x, \epsilon, \delta_{\epsilon}}\right) \mathrm{d} r\right\},\right.
\end{aligned}
$$

then we deduce that $u^{\epsilon, \delta_{\epsilon}}(t, x)>0$.
We have the

Theorem 3.3 For $(t, x) \in] 0,+\infty\left[\times \operatorname{IR}^{\mathrm{d}}\right.$, we have 1)

$$
\begin{aligned}
& \lim _{\epsilon \downarrow 0} \epsilon \log u^{\epsilon, \delta_{\epsilon}}(t, x)=V^{*}(t, x) \\
& =\inf _{\Theta_{t}}\left\{\bar{C} \tau-\inf S_{0 \tau}(\varphi), \varphi_{0}=x, \varphi_{t} \in G_{0}, \varphi \in \mathcal{C}([0, t])\right\} .
\end{aligned}
$$

2)

$$
\lim _{\epsilon \downarrow 0} u^{\epsilon, \delta_{\epsilon}}(t, x)=0,
$$

uniformly in any compact set $\mathcal{K}$ of $\mathcal{E}$.

3)

$$
\lim _{\epsilon \downarrow 0} u^{\epsilon, \delta_{\epsilon}}(t, x)=1,
$$

in all compact set $\mathcal{K}^{\prime}$ of $\stackrel{\circ}{\mathcal{M}}$.

Proof: For first item, the proof is the same as in [2].

For the second point we can see that there exists $C>0$ such that

$$
0 \leq u^{\epsilon, \delta_{\epsilon}}(t, x)<\mathrm{e}^{-\frac{C}{\epsilon}}, \forall(t, x) \in \mathcal{K} .
$$

The third item is an immediate consequence of 1 ).

\section{REFERENCES}

[1] D. Nualart, "The Malliavin Calculus and Related Topics. Probability and Its Applications," Springer-Verlag, New York, 1995

[2] A. Diédhiou and C. Manga, "Application of Homogeneization and Large Deviations to a Parabolic Semilinear Equation," Journal of Mathematical Analysis and Applications, Vol. 342, No. 1, 2008, pp. 146-160.

[3] M. I. Freidlin and R. B. Sowers, "A Comparison of Homogenization and Large Deviations, with Applications to Wavefront Propagation," Stochastic Processes and Their Applications, Vol. 82, No. 1, 1999, pp. 23-32. doi:10.1016/S0304-4149(99)00003-4

[4] É. Pardoux and S. Peng, "Backward Stochastic Differential Equations and Quasi-Linear Parabolic Differential Equations," In: B. L. Rozovskii and R. B. Sowers, Eds., Stochastic Partial Differential Equations and Their Applications, Lecture Notes in Control and Information Sciences, Vol. 176, 1992, pp. 200-217. doi:10.1007/BFb0007334

[5] A. Diédhiou and É. Pardoux, "Homogenization of Periodic Semilinear Hypoelliptic PDES," Annales de la faculté des sciences de Toulouse Mathématiques, Vol. 16, No. 2, 2007, pp. 253-283.

[6] É. Pardoux, "Homogenization of Linear and Semilinear Second Order Parabolic PDEs with Periodic Coefficients: A Probabilistic Approch," Journal of Functional Analysis, Vol. 167, No. 2, 1999, pp. 498-520. doi:10.1006/jfan.1999.3441

[7] É. Pardoux, "BSDEs, Weak Convergence and Homogenization of Semilinear PDEs," In: F. H. Clarke and R. J. 
Stern, Eds., Nonlinear Analysis, Differential Equations and Control, Springer, Berlin, 1999, pp. 503-549.

[8] A. Dembo and O. Zeitouni, "Large Deviations Tech- niques and Applications," Jones and Bartlett, Boston, 2010 . 